\title{
BMJ Open Identifying patient characteristics associated with potentially redirectable paramedic transported emergency department visits in Ontario, Canada: a population-based cohort study
}

\author{
Ryan P Strum (D) , 'Walter Tavares, ${ }^{2}$ Andrew Worster, ${ }^{1,3}$ Lauren E Griffith (1) , ${ }^{1,4}$ \\ Andrew P Costa (1) ${ }^{1,5}$
}

To cite: Strum RP, Tavares W, Worster A, et al. Identifying patient characteristics associated with potentially redirectable paramedic transported emergency department visits in Ontario, Canada: a population-based cohort study. BMJ Open 2021;11:e054625. doi:10.1136/ bmjopen-2021-054625

- Prepublication history for this paper is available online. To view these files, please visit the journal online (http://dx.doi. org/10.1136/bmjopen-2021054625).

Received 21 June 2021 Accepted 01 December 2021

Check for updates

(C) Author(s) (or their employer(s)) 2021. Re-use permitted under CC BY-NC. No commercial re-use. See rights and permissions. Published by BMJ.

For numbered affiliations see end of article.

Correspondence to

Ryan P Strum;

strumr@mcmaster.ca

\section{ABSTRACT}

Objective Paramedic redirection from emergency department (ED) to subacute centres may be more beneficial for some patients, though little is known about which patients are potentially appropriate. We examined whether patient characteristics were associated with ED visits when the main intervention was suitable to be performed in a subacute centre.

Methods We conducted a retrospective observational study using the National Ambulatory Care Reporting System from 2014 to 2018 in Ontario, Canada. We included all adult patients transported by paramedics and had a main physician intervention recorded. We used results of a RAND/UCLA modified Delphi study to categorise patients into either ED or a subacute care (urgent care and/or general practice centre) based on their main intervention. An independent logistic regression model was analysed for each subacute centre.

Results A total of 2394072 ED visits were included; $59 \%$ of ED interventions were categorised as 'urgent care', $27 \%$ 'ED only', $9 \%$ either 'urgent care' or 'general practice' and $5 \%$ had an intervention not previously classified. ED visits suitable for 'general practice' had the highest percentage of patients discharged, while 'ED only' had the lowest. Lower medical acuity, younger age, time of triage in evening and overnight, and discharged from ED were independently associated with both subacute centres. 'Urgent care' visits/interventions were associated with an ED main diagnosis of the respiratory system (OR 3.49), while 'general practice' visits were associated with mental health disorders (OR 9.85) and injury/poison/consequences of external causes (OR 3.38).

Conclusions The majority of ED visits had a main intervention that could have potentially been conducted in a subacute centre. We identified characteristics and diagnostic patterns associated with $E D$ visits when the main intervention was categorised as a subacute centre intervention. This study contributes knowledge to inform which patients are potentially appropriate for paramedic redirection.
Strengths and limitations of this study

- This study is the first to describe and examine which patient characteristics were associated with paramedic transported emergency department (ED) visits when the main intervention was suitable to be conducted a subacute care centre.

- Our study included all adult ED visits transported by paramedics in Ontario, Canada from 1 January 2014 to 31 March 2018, constituting a population-based cohort.

- ED visits were classified into three categories based on where the main intervention potentially could be conducted: 'ED only', 'urgent care' and 'general practice'.

- Patient characteristic associations were investigated using unadjusted and adjusted binary logistic regression.

Due to inherent limitations of administrative database recording, we could only include visit characteristics from records when the main physician intervention field was recorded.

\section{BACKGROUND}

Patients with non-emergent medical conditions constitute the majority of paramedic emergency department (ED) transports in Ontario, Canada. ${ }^{1}$ Contrary to traditional paramedic service delivery models, $60 \%$ of transported patients have non-emergent medical acuities and half $(51 \%)$ are categorised as 'urgent' (Canadian Triage and Acuity Scale, CTAS). ${ }^{12}$ Usage of paramedic services have broadened and increased to provide access and transport to healthcare by patients that do not necessarily require acute management, thereby overcrowding ED's and increasing workloads. ${ }^{34}$ The most appropriate setting may not be the ED when visits are non-emergent, and have complex primary care needs or require greater assessment 
times. ${ }^{15}$ Subacute care centres could offer similar or more efficient care alternatives for non-emergent patients and at a higher cost effectiveness, though paramedics are restricted from ED transport deviation. ${ }^{1267}$

The evidence for safe paramedic redirection from ED to subacute centres is established in North America and internationally, though the literature for relative effectiveness is inconclusive. ${ }^{8-12}$ A major limitation has been the lack of consistent targeting of which patients could be appropriately redirected to alternative care, and which cannot. No patient classification system has been developed or implemented to evaluate patient suitability for redirection. ${ }^{13-15}$ To that end, we conducted a RAND/ UCLA modified Delphi study to evaluate ED main intervention applicability for their suitability to be conducted in three subacute centres. ${ }^{116}$ However, the characteristics of the ED visits determined appropriate for subacute has not been reported. Incorporating patient characteristics (ie, age, acuity, diagnosis, ED visit outcome) associated with ED visits suitable for subacute centres dependent on the main visit intervention will be important to further contextualise an epidemiological classification of which patients are potentially redirectable. ED visit main interventions performed by physicians could be a core component to classifying patient suitability for redirection that has been overlooked in previous categorisation of which patients are potentially appropriate ${ }^{13-15}$ Inclusion of ED main interventions has the potential to scaffold with additional characteristic variables to construct a robust epidemiological patient classification for potentially identifying redirectable visits for paramedics.

Our objective was to examine which patient characteristics were associated with $\mathrm{ED}$ visits that had a recorded main intervention suitable to be conducted in a subacute care centre in a population-based cohort from Ontario, Canada. We hypothesised that younger patient age, lower medical acuity and being discharged from ED as the visit outcome would be associated with ED visits with a main intervention suitable to be conducted in subacute care centres.

\section{METHODS}

\section{Design}

We used a retrospective observational design to analyse secondary administrative ED patient records from the National Ambulatory Care Reporting System (NACRS) database. The Strengthening the Reporting of Observational Studies in Epidemiology statement was followed for reporting this study. ${ }^{17}$

\section{Population}

All Ontario adult patients ( $\geq 18$ years) transported to the ED by paramedics following a 911 request and had a recorded triage acuity score were eligible. Visits were excluded if they did not have a recorded main physician intervention in NACRS, as visit could not be classified into a care centre. We excluded any individual who was not triaged by hospital staff (registered but left prior to triage) or was not assigned a CTAS. Sampling methods were not incorporated as all ED visits satisfying eligibility criteria were included as to minimise potential bias.

\section{Data sources}

This study used ED NACRS data from 1 January 2014 to 31 March 2018, representing the most recently available records at time of study initiation. NACRS is a hospital and community-based ambulatory care administrative database that collects patient visit data at the time of service. ${ }^{1819}$ All hospitals in Ontario are mandated to submit electronic patient abstracts from the ED to comply with standard reporting or quality control measures. NACRS was accessed through the Institute for Clinical Evaluative Sciences (IC/ES) Data \& Analytical Virtual Environment (IDAVE) portal. Briefly, IC/ES is a non-profit, independent corporation that supports the study of health service and population-wide outcomes in Ontario using administrative databases. IDAVE acts as a secure virtual server for researchers to access approved cohort created data for scientific research and manuscript-ready output.

\section{Variables and codification}

We used a RAND/UCLA modified Delphi design to assess the 150 most frequently recorded main physician intervention codes for conduction in the ED exclusively, or in any of three subacute care centres (urgent care centre, walk-in medical centre and/or nurse practitioner-led clinic). ${ }^{16}$ Modified Delphi studies use an iterative process to systematically examine the collective consensus of an expert group through repeated rounds of individual ratings. ${ }^{20}$ This methodology constituted a reliable strategy to determine consensus on defined clinical problems where there is little or no effective evidence with high internal validity. ${ }^{212}$ Consensus was achieved on 146 interventions; 43 were rated for 'ED only', 103 for 'urgent care', 46 for 'walk-in medical centres' and 47 for 'nurse practitioner-led clinics'. ${ }^{16}$ Walk-in medical centres and nurse practitioner-led clinics had high similarity in results with 44 interventions in common. Due to high agreement, these centres were collapsed into a category called 'general practice' and included all interventions from either centre. ED visits were sorted into three categories for study, based on the consensus of this previous classification of patient main interventions: 'ED only', 'urgent care' and 'general practice'. ED visits categorised as 'general practice' were also categorised for 'urgent care', thus 'general practice' represents a specific subset of the 'urgent care' cohort.

Patient characteristics selection was based on clinical judgement, scientific literature and access availability to variables. Age was grouped into twenty ordinal levels by IC/ES due to privacy restrictions, and further collapsed into three categories for grouping of similar patients in similar life-stage progressions $(18-39,40-64,65-105$ years). Access to primary care is assigned as the physician class that provides the patients usual source of care prior 
to $\mathrm{ED}$ visit. Triage acuity was assigned to patients by the ED triage nurse on entry to ED using CTAS. CTAS is an ordinal scale that ranges from one to five, with a score of one to act as the most emergent (resuscitation) and five as least urgent (non-urgent) ${ }^{23}$ Triage acuity was collapsed into three categories (emergent, urgent, non-urgent) to ensure model stability as relatively few patients receive a CTAS score of one $(4.4 \%)$ or five $(3.2 \%)$. ED main diagnosis was assigned by the attending ED physician and recorded using the International Statistical Classification of Diseases and Related Health Problems, 10th revision (ICD-10). ICD-10 is recognised as the international standard for reporting diagnostic conditions, and managed by the WHO internationally, and Canadian Institute of Health Information in Canada. ${ }^{24}$ ICD-10 diagnostic codes were collapsed into eight categories to ensure model stability; diagnostic categories comprising less than $5 \%$ of the study cohort were grouped together.

\section{Statistical analysis}

Analyses of patients visit characteristics were described using general measures of frequency. Association of characteristics to each subacute care centre was examined using two separate binary logistic regression analyses to make distinctions between visits/interventions suitable for 'urgent care' and 'general practice'. Only ED visits with main interventions classified in the RAND/UCLA modified Delphi study were included in the modelling analyses; visits with interventions not classified were excluded. Results were reported using unadjusted and adjusted ORs for each model with corresponding 95\% CIs. Data were managed and analysed in R software, V.3.4. All variables used in the models were reported with a significantly high completion rate $(>99 \%)$. For this reason, as required, data were directly stated as unreported or missing where applicable; imputation was not required.

\section{Patient and public involvement}

Front-line paramedics in Canada were consulted to gauge their satisfaction with the reporting and potential implications of this study. The reporting of results received input from paramedic services in several provinces across Canada. All input helped to modify the study design, analysis plan and preparation of the manuscript.

\section{RESULTS}

\section{Descriptive statistics}

This study included 2394072 adult patients transported by paramedics to an Ontario ED and had a main physician intervention recorded in NACRS. The study cohort represents $68.5 \%$ of all $\mathrm{ED}$ visits transported by paramedics in the study period (2 394 072/3 493 059). All ED visits absent of a recorded main intervention were excluded. In this cohort, $59 \%$ of ED visits were categorised as having a main intervention suitable to be conducted in 'urgent care', 27\% were 'ED only', 9\% either 'urgent care' or 'general practice', and 5\% did not have an intervention

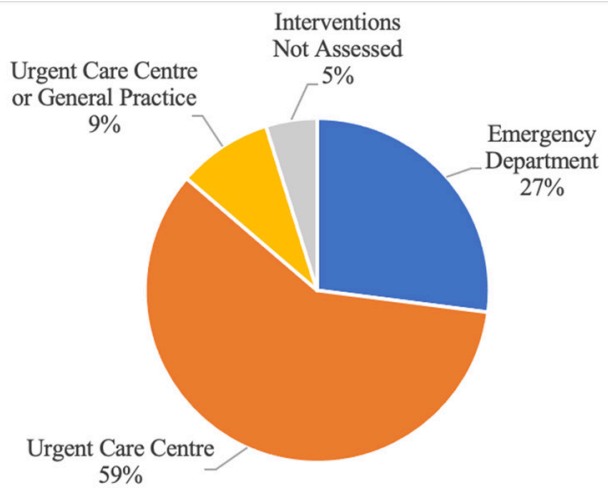

Figure 1 Breakdown of ED visits where the main physician intervention could potentially be conducted based on care setting. The cohort represents all Ontario paramedic transported ED visits when the main intervention was recorded in the National Ambulatory Care Reporting System database. ED, emergency department.

classified in the RAND/UCLA modified Delphi study. The proportion of patients sorted into care settings based on categorised main intervention consensus is shown in figure 1 .

To facilitate the comparison of characteristics among the three care categories, the $5 \%$ of ED visits with recorded main physician interventions but not classified in the RAND/UCLA modified Delphi study were excluded from the following descriptive statistics and modelling analyses. A total of $2267585 \mathrm{ED}$ visits $(94.7 \%$ of the original cohort) were included in the following analyses.

Of ED visit interventions classified in the RAND/ UCLA modified Delphi study, most were suitable for 'urgent care' $(68.7 \%)$, of which some were also fitting for 'general practice' (10.3\%); interventions classified as 'ED only' comprising the rest (31.3\%). All ED visits with interventions classified suitable for 'general practice' centres were also suitable for 'urgent care' centres. Visit interventions suitable for 'general practice' had the highest proportion of visits in the youngest age category $(29.7 \%)$, while visits classified as 'ED only' had the highest proportion in the oldest age category (63.6\%). Across the three centres classified, triage acuities two and three were the largest CTAS proportions. Of main diagnosis categorisations, visits classified for 'ED only' had the largest patient proportion with diseases in the nervous, circulatory and digestive systems. 'Urgent care' classified ED visits had the largest proportion in infectious diseases, endocrine/nutrition/metabolic disorders, respiratory system and diseases of the musculoskeletal system. ED visits classified for 'general practice' had the largest in mental/behavioural disorders and injury/poisoning/ certain other consequences of external causes. Missing data were not found for most included characteristics, however minimally in access to primary care $(5.0 \%)$. The characteristics of the study cohort are shown in table 1.

All three classified centres showed increases in proportion of ED visits discharged as CTAS scoring increased 
Table 1 Characteristics of the study cohort assessed in the ED, grouped by healthcare setting that could conduct the main ED intervention

\begin{tabular}{|c|c|c|c|}
\hline Characteristic & ED only, no. (\%) & Urgent care centre, no. (\%) & General practice, no. (\%)* \\
\hline Overall, $2,267,585$ & $710340(31.3) \dagger$ & $1557245(68.7) \dagger$ & $233896(10.3) \dagger$ \\
\hline \multicolumn{4}{|l|}{ Gender } \\
\hline Male & $335526(47.2)$ & $701921(45.1)$ & $118624(50.7)$ \\
\hline Female & $374814(52.8)$ & $855324(54.9)$ & $115272(49.3)$ \\
\hline \multicolumn{4}{|l|}{ Age, years } \\
\hline $18-39$ & $68456(9.6)$ & $222126(14.3)$ & $69550(29.7)$ \\
\hline $40-64$ & $187776(26.4)$ & $407518(26.2)$ & 70409 (30.1) \\
\hline $65-105$ & $452013(63.6)$ & 927601 (59.6) & 93937 (40.2) \\
\hline \multicolumn{4}{|l|}{ Access to primary health care } \\
\hline Family physician & $628410(88.5)$ & $1380558(88.7)$ & $190855(81.6)$ \\
\hline Other & $3070(0.4)$ & $10446(0.7)$ & $2875(1.2)$ \\
\hline None & $45255(6.4)$ & $102792(6.6)$ & $24804(10.6)$ \\
\hline Unreported & $33605(4.7)$ & $63449(4.1)$ & $15362(6.6)$ \\
\hline \multicolumn{4}{|l|}{ Year of visit } \\
\hline 2014 & $142988(20.1)$ & $360483(23.1)$ & 60677 (25.9) \\
\hline 2015 & $157724(22.2)$ & $361686(23.2)$ & $54942(23.5)$ \\
\hline 2016 & 172327 (24.3) & 365899 (23.5) & $53767(23.0)$ \\
\hline 2017 & $187015(26.3)$ & $368925(23.7)$ & 52401 (22.4) \\
\hline 2018 & $50286(7.1)$ & $100252(6.4)$ & $12109(5.2)$ \\
\hline \multicolumn{4}{|l|}{ CTAS, acuity } \\
\hline 1-Resuscitation & $55787(7.9)$ & $37559(2.4)$ & $6067(2.6)$ \\
\hline 2-Emergent & $310363(43.7)$ & $611786(39.3)$ & 85448 (36.5) \\
\hline 3Urgent & $323284(45.5)$ & $789010(50.7)$ & $116014(49.6)$ \\
\hline 4-Less Urgent & $20108(2.8)$ & $113891(7.3)$ & $25020(10.7)$ \\
\hline 5-Non-urgent & $798(0.1)$ & $4999(0.3)$ & $1347(0.6)$ \\
\hline \multicolumn{4}{|l|}{ Time of triage, hour } \\
\hline Day (0700 -1459) & $319312(45.0)$ & $652013(41.9)$ & 81448 (34.8) \\
\hline Evening (1500-2259) & $264375(37.2)$ & $593972(38.1)$ & $95383(40.8)$ \\
\hline Overnight (2300-0659) & $126653(17.8)$ & $311260(20.0)$ & $57065(24.4)$ \\
\hline \multicolumn{4}{|l|}{ Diagnostic Category, ICD-10‡ } \\
\hline A,B-Certain infectious diseases & $17995(2.5)$ & $50360(3.2)$ & $2904(1.2)$ \\
\hline C-Neoplasms & $8429(1.2)$ & $10781(0.7)$ & $653(0.3)$ \\
\hline $\begin{array}{l}\text { D-Disorders of blood involving immune } \\
\text { system }\end{array}$ & $4250(0.6)$ & $11692(0.8)$ & $927(0.4)$ \\
\hline $\begin{array}{l}\text { E-Endocrine, nutrition, and metabolic } \\
\text { disorders }\end{array}$ & $13805(1.9)$ & $34167(2.2)$ & $3249(1.4)$ \\
\hline F-Mental and behavioural disorders & $35363(5.0)$ & $86911(5.6)$ & $57728(24.7)$ \\
\hline G-Diseases of nervous system & $43001(6.1)$ & $9912(0.6)$ & $2658(1.1)$ \\
\hline $\begin{array}{l}\mathrm{H} \text {-Diseases of the eye, adnexa, ear and } \\
\text { mastoid process }\end{array}$ & $9132(1.3)$ & $2329(0.1)$ & $698(0.3)$ \\
\hline I-Diseases of the circulatory system & $80452(11.3)$ & $130083(8.4)$ & $7585(3.2)$ \\
\hline $\mathrm{J}$-Diseases of the respiratory system & $34828(4.9)$ & $214284(13.8)$ & $13735(5.9)$ \\
\hline K-Diseases of the digestive system & $44821(6.3)$ & $76472(4.9)$ & $5074(2.2)$ \\
\hline $\begin{array}{l}\text { L-Diseases of the skin and subcutaneous } \\
\text { tissue }\end{array}$ & $2188(0.3)$ & $14220(0.9)$ & $998(0.4)$ \\
\hline
\end{tabular}




\begin{tabular}{|c|c|c|c|}
\hline Characteristic & ED only, no. (\%) & Urgent care centre, no. (\%) & General practice, no. $(\%)^{\star}$ \\
\hline $\begin{array}{l}\text { M-Diseases of the musculoskeletal system } \\
\text { and connective tissue }\end{array}$ & $16385(2.3)$ & $75194(4.8)$ & $2515(1.1)$ \\
\hline $\mathrm{N}$-Diseases of the genitourinary system & $36992(5.2)$ & $69707(4.5)$ & $5558(2.4)$ \\
\hline O-Pregnancy, childbirth, and the puerperium & $195(0.0)$ & $2171(0.1)$ & $202(0.1)$ \\
\hline $\begin{array}{l}\text { P-Certain conditions origination in the } \\
\text { Perinatal Period }\end{array}$ & $2(0.0)$ & $1(0.0)$ & $0(0.0)$ \\
\hline $\begin{array}{l}\text { Q-Congenital malformations, deformations } \\
\text { and chromosomal abnormalities }\end{array}$ & $119(0.0)$ & $115(0.0)$ & $19(0.0)$ \\
\hline $\begin{array}{l}\text { R-Symptoms, signs and abnormal clinical and } \\
\text { laboratory findings }\end{array}$ & $198556(28.0)$ & $376542(24.2)$ & $39005(16.7)$ \\
\hline $\begin{array}{l}\text { S,T-Injury, poisoning and certain other } \\
\text { consequences of external causes }\end{array}$ & $152591(21.5)$ & $370165(23.8)$ & $87161(37.3)$ \\
\hline U-External coucals of morbidity and mortality & $0(0.0)$ & $2(0.0)$ & $0(0.0)$ \\
\hline $\begin{array}{l}\text { Z-Factors influencing health status and } \\
\text { contact with health services }\end{array}$ & $11236(1.6)$ & $22137(1.4)$ & $3227(1.4)$ \\
\hline $\begin{array}{l}\text { Visit outcome admitted to reporting facility, to } \\
\text { special }\end{array}$ & $44651(6.3)$ & $49851(3.2)$ & $3950(1.7)$ \\
\hline \multicolumn{4}{|l|}{ Care unit or operating room } \\
\hline Admitted to reporting facility, to another unit & 283378 (39.9) & $541719(34.8)$ & $51593(22.1)$ \\
\hline Discharged home & $291956(41.1)$ & $773555(49.7)$ & $142624(61.0)$ \\
\hline Discharged to place of residence (Institution) & $62062(8.7)$ & $143350(9.2)$ & 24128 (10.3) \\
\hline Dead on or after arrival in ED & $540(0.1)$ & $441(0.0)$ & $32(0.0)$ \\
\hline Other & $27753(3.9)$ & 36669 (2.4) & $11569(4.9)$ \\
\hline \multicolumn{4}{|l|}{ Days to Left ED } \\
\hline 0 & $416687(58.7)$ & $1012242(65.0)$ & 162894 (69.6) \\
\hline 1 & 261869 (36.9) & 487555 (31.3) & 66194 (28.3) \\
\hline 2 & 25725 (3.6) & $46228(3.0)$ & $3743(1.6)$ \\
\hline 3 & $4833(0.7)$ & $9013(0.6)$ & $799(0.3)$ \\
\hline 4 & $820(0.1)$ & $1628(0.1)$ & $175(0.1)$ \\
\hline$>4$ & $231(0.0)$ & $286(0.0)$ & $91(0.0)$ \\
\hline
\end{tabular}

${ }^{*}$ General practice is a subset of urgent care; visits categorised as general practice were also categorised as urgent care. †Percentage of the total patients in cohort.

$\ddagger$ Represents primary diagnosis of ED visit.

CTAS, Canadian Triage and Acuity Score; ED, emergency department; ICD-10th, International Statistical Classification of Diseases, 10th revision.

from one to four (ie, medical acuity decreased), with CTAS four to five holding generally consistent. ED visits classified as 'urgent care' were similar in discharge percentages with 'ED only' visits for CTAS scores one and two $(17.4 \%$ vs $13.5 \%$; 50.0 vs $46.5 \%)$, but similar in discharge percentages with classified visits for 'general practice' in higher CTAS scores four and five $(81.5 \%$ vs $89.0 \%, 79.6 \%$ vs $86.3 \%$ ). There was no overlap among the three care centre categories in percentage of ED visits discharged across all CTAS levels. A visual representation of ED visits by care centre and acuity is shown in figure 2 .

\section{Main results}

In an adjusted model, ED visits/interventions classified suitable for 'urgent care' were significantly associated with urgent and non-urgent triage acuities (OR 1.38, $95 \%$ CI 1.37 to 1.39 ; OR $3.10,95 \%$ CI 3.05 to 3.15 ), evening and overnight triage times (OR 1.10, 95\% CI 1.09 to 1.11 ; OR $1.16,95 \%$ CI 1.15 to 1.17 ) and main diagnoses of the respiratory system (OR $3.49,95 \%$ CI 3.44 to 3.54 ) and mental health disorders (OR 1.06, 95\% CI 1.04 to 1.08$)$. Visits classified for 'urgent care' had a $26 \%$ odds reduction in hospital admission compared with 'ED only' classified visits (OR $0.74,95 \%$ CI 0.73 to 0.74 ). Additionally, older age groups $(40-64,65-105)$ had reduced odds of association with 'urgent care'. The area under the receiver operating characteristic curve (AUC) was 0.625, inferring this adjusted model is a less than fair classifier to identify patient visit associations with 'urgent care'. 


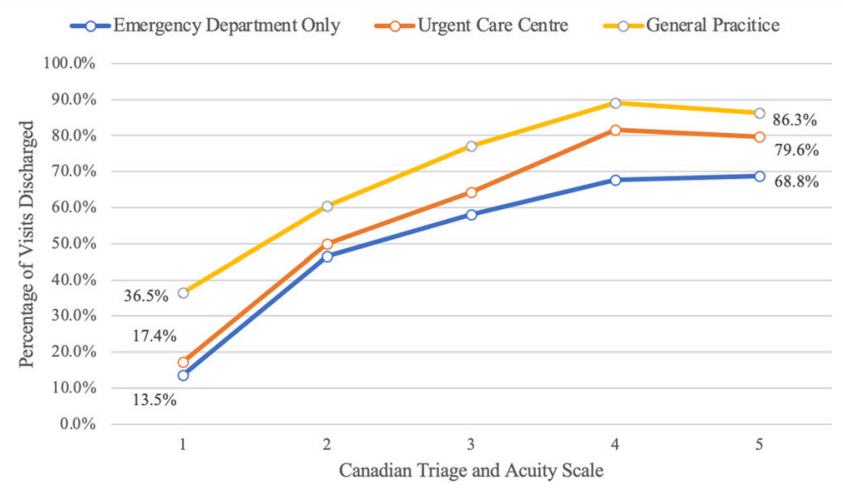

Figure 2 Percentage of ED visits discharged from unit, broken down triage acuity and healthcare centre classified by the main physician intervention. ED, emergency department.

In a second adjusted model, ED visits/interventions classified suitable for 'general practice' also had significant associations with urgent and non-urgent triage acuities (OR 1.49, 95\% CI 1.47 to 1.51 ; OR $4.40,95 \%$ CI 4.30 to 4.50$)$. ED main diagnoses of the digestive system (OR $1.10,95 \%$ CI 1.05 to 1.14 ), genitourinary system (OR $1.15,95 \%$ CI 1.11 to 1.20 ), mental health disorders (OR 9.85, 95\% CI 9.56 to 10.14), symptoms and signs of abnormal clinical labs (OR 1.38, 95\% CI 1.34 to 1.42 ) and injury consequences of external causes (OR 3.38, 95\% CI 3.29 to 3.48 ) were all significantly associated with visits/ interventions suitable for 'general practice' centres. ED visits classified for 'general practice' had a $49 \%$ odds reduction in hospital admission compared with 'ED only' (OR $0.51,95 \%$ CI 0.50 to 0.52 ). Older age groups of 40-64 years and 65-105years had reducing odds of association (OR $0.50,95 \%$ CI 0.49 to 0.50 ; OR $0.32,95 \%$ CI 0.32 to 0.33 ). This adjusted model was a fair classifier to identify patient visit associations with an AUC of 0.772 for 'general practice'. Table 2 shows the unadjusted and adjusted models for patient characteristic associations with subacute care settings in comparison with interventions classified as 'ED only'.

\section{Interpretation}

The majority of ED visits with a recorded main intervention were suitable to have the intervention conducted in an urgent care centre. Similar characteristics were found in both subacute centres in the younger age groups, lower triage acuity's, triage times not during day hours, and discharged from ED as the visit outcome. The subacute care groups differed in associated characteristics of gender and main diagnoses. 'Urgent care' was positively associated with the female gender, and diagnoses of the respiratory system and mental health disorders. Visits classified for 'general practice' were positively associated with the male gender, and main diagnoses of the digestive system, genitourinary system, mental health disorders, abnormal clinical labs, injuries of external causes and the remaining diagnostic categories collapsed together. Our hypothesis of patient characteristics associations was accurate and supported in both models.

Our study that examines the associated characteristics of ED visits that may be suitable for subacute centres is novel, given there is no previous literature directly comparable. The most analogous literature resides in articles that studied characteristic associations of patients with low acuity ED visits. Our study yielded similar results to the literature, finding patients triaged in the evening and overnight hours, and having a primary complaint categorisation of psychiatric or toxicology/poisoning were correlated with non-emergent ambulance usage for ED transportation. ${ }^{25}$ While some literature suggests that older age is associated with non-emergent paramedic transported visits, our study contrasts this finding with both subacute centres being associated with the youngest group (18-39years). ${ }^{25-27}$ The percentage of ED visits appropriate for general practice settings was $10.3 \%$ of the study cohort, a consistent result with studies that have estimated this range to be $10 \%-12 \% .{ }^{14}$ Visits discharged from the ED classified as 'urgent care' or 'general practice' is consistent with previous analyses of paramedic transported ED visits that have low priority conditions. ${ }^{2}$

In this study, patient visit characteristics were identified for their independent association with two subacute care centre classifications compared with 'ED only' visits based on suitability of their main intervention. Several associated characteristics were consistent between the two subacute classifications, but some differences were recognised in diagnostic categories. These differences may be due to the much larger number and variation of ED interventions identified by experts as potentially appropriate for urgent care (103) than for general practice (47), and corresponding larger visit incidence of each centre (1557245 urgent care, 233896 general practice). Mental health as a main diagnostic category was associated significantly with classification of 'general practice' compared with 'ED only' (OR 9.85, 95\% CI 9.56 to 10.14). All included mental health interventions were suitable for subacute care, and none exclusively for the ED, which may explain this finding. Although not represented in the RAND/UCLA modified Delphi study, some mental health patients may benefit from transport to facilities that can provide specific mental healthcare that is not a general practice centre. No cross-over in percentage of patients discharged was observed among the centre classifications at each ordinal CTAS level, inferring a plausible hierarchical relationship may exist in ability to manage acuity.

Identification of characteristics associated with paramedic transported patients that could have potentially received their main intervention in subacute care will help to inform further study of out-of-hospital redirection classifications. Two-thirds of patients that visited the ED could have potentially received their intervention in an urgent care centre, though paramedics are restricted from urgent care transport and are relatively rare compared with other subacute alternatives. This study serves as a 
Table 2 Unadjusted and adjusted patient visit characteristics associated with urgent care and general practice setting interventions, compared against ED only intervention

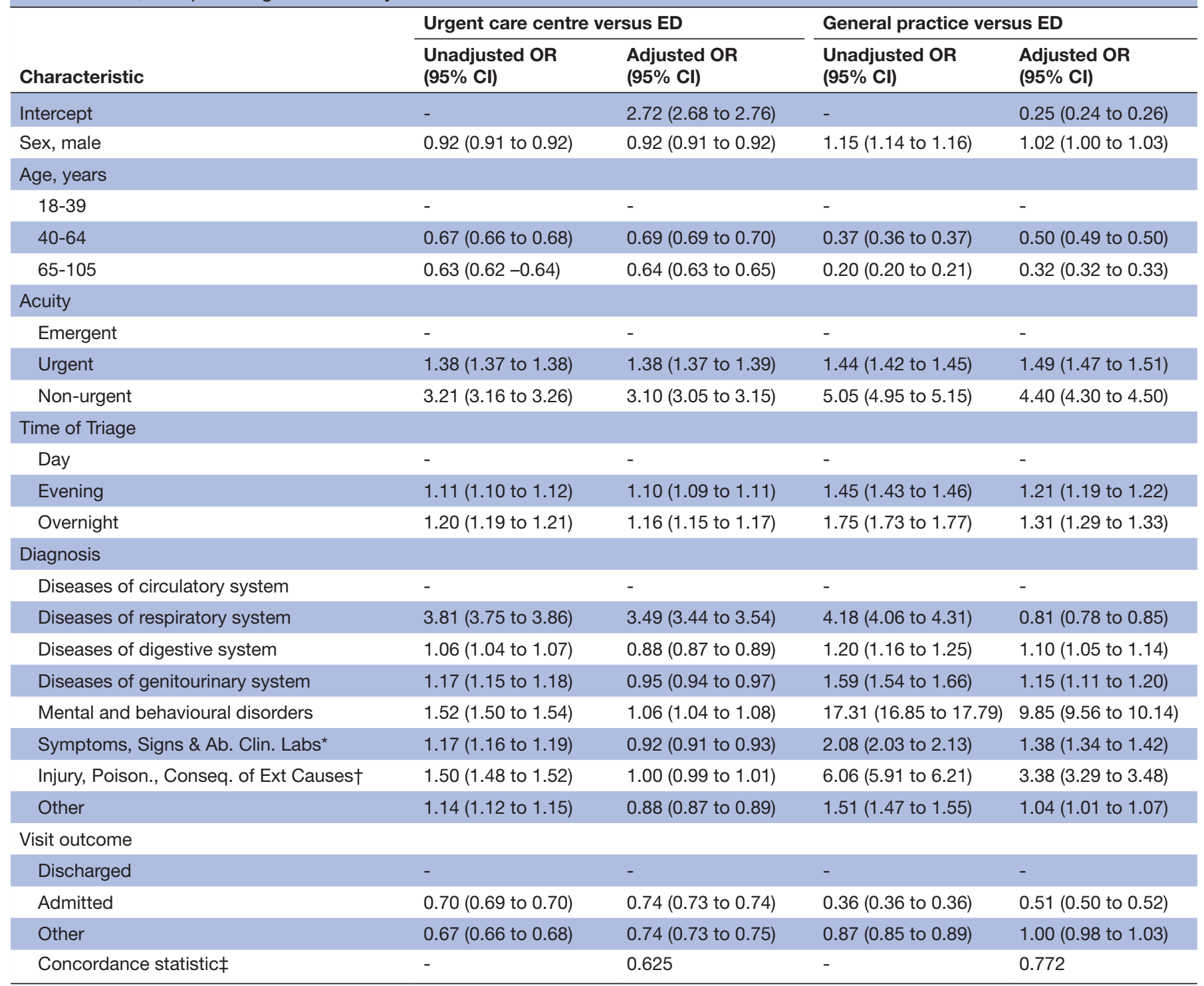

*Symptoms, signs and abnormal clinical and laboratory findings.

†Injury, poisoning and certain other consequences of external causes.

†Reported as area under the receiver operating characteristic curve $(95 \% \mathrm{Cl})$.

ED, emergency department.

first step to constructing and describing an epidemiological patient classification, however these results cannot be translated into clinical guidance without prospective validation. Future research is required to provide additional contextualisation of patients, especially patients admitted from ED that were determined as appropriate for subacute centres. The patient characteristics identified in this study will aid in classifying which characteristics should be included in a high specific patient classification system to examine retrospectively which patients may have been suitable for paramedic. Development of paramedic redirection protocols can be supported by this research to inform potential patient eligibility criteria, though further evidence must be incorporated prior to integrating these results into paramedic clinical practices, such as non-clinical features, operational features, patient values/preferences and external validation research. The results of this study will contribute novel evidence to paramedic-based programmes that intend to support ED redirection protocols or research, independent of healthcare insurance structures. ${ }^{128}$

\section{Limitations}

Given the inherent limitations of ED administrative databases in a retrospective observational design, we could only determine the characteristics of ED visits based on the completeness of the main physician intervention field. Our dataset of NACRS contained $68.5 \%$ of all recorded ED visits in Ontario, of which this study's analyses examined $94.7 \%$ of these visits as they were previously classified 
in a RAND/UCLA modified Delphi study (2 267 585). A proportion of missing data in recorded ED interventions is expected in administrative datasets; some admitted patients may have had their ED interventions recorded in the Discharge Abstract Database as opposed to NACRS, or in some instances there is no intervention or has not been recorded. This study used the results of a modified Delphi consensus exercise to categorise patients, a methodology that has its own inherent limitations, though were minimised. Lastly, this study was pursued for epidemiological purposes using a specific population (adult, paramedic transported) and cannot inform clinical decision management without further patient contextualisation or prospective testing.

\section{CONCLUSION}

Paramedic redirection of some non-emergent patients to alternative subacute care could be a pragmatic strategy to improve patient-centred care (ie, by better aligning paramedic services with patient needs) and ED utilisation in North America. Categorisation of patients into the most appropriate care centre based on RAND/UCLA modified Delphi panel consensus allowed for study of patient characteristics associated with 'urgent care' and 'general practice' centres. This epidemiological study will provide evidence and knowledge to inform construction of a patient classification to potentially redirectable paramedic transported patients, and augment further research in paramedic alternative destination protocols.

\section{Author affiliations}

${ }^{1}$ Health Research Methods, Evidence, and Impact, McMaster University, Hamilton, Ontario, Canada

${ }^{2}$ The Wilson Centre and Post MD Education, University of Toronto Faculty of Medicine, Toronto, Ontario, Canada

${ }^{3}$ Division of Emergency Medicine, McMaster University, Hamilton, Ontario, Canada ${ }^{4}$ McMaster Institute for Research and Aging, McMaster University, Hamilton, Ontario, Canada

${ }^{5}$ Department of Medicine, McMaster University, Hamilton, Ontario, Canada

\section{Twitter Ryan P Strum @Ryan_Strum and Lauren E Griffith @LaurenGriff1}

Contributors RPS and APC led the conceptualisation of the study objective and methodology. RPS designed the study, conducted the analysis, and drafted and revised the manuscript. WT, AW and LEG made contributions to the methodology and interpretation. All authors critically revised the manuscript, approved the final version and agreed to act as guarantors.

Funding The authors have not declared a specific grant for this research from any funding agency in the public, commercial or not-for-profit sectors.

Competing interests None declared.

Patient and public involvement Patients and/or the public were involved in the design, or conduct, or reporting, or dissemination plans of this research. Refer to the Methods section for further details.

Patient consent for publication Not applicable.

Ethics approval IC/ES's collection of ambulatory care ED administrative data were authorised under Section 45 of the Personal Health Information Protection Act, which does not require review by a Research Ethics Board; all data were absent of personal health identifying information.

Provenance and peer review Not commissioned; externally peer reviewed.

Data availability statement All data relevant to the study are included in the article.
Open access This is an open access article distributed in accordance with the Creative Commons Attribution Non Commercial (CC BY-NC 4.0) license, which permits others to distribute, remix, adapt, build upon this work non-commercially, and license their derivative works on different terms, provided the original work is properly cited, appropriate credit is given, any changes made indicated, and the use is non-commercial. See: http://creativecommons.org/licenses/by-nc/4.0/.

ORCID iDs

Ryan P Strum http://orcid.org/0000-0003-1902-4734

Lauren E Griffith http://orcid.org/0000-0002-2794-9692

Andrew P Costa http://orcid.org/0000-0001-9212-5641

\section{REFERENCES}

1 Strum RP, Tavares W, Worster A, et al. Development of the PriCARE classification for potentially preventable emergency department visits by ambulance: a RAND/UCLA modified Delphi study protocol. BMJ Open 2021:11:e045351.

2 Tavares W, Drennan I, Van Diepen K, et al. Building capacity in healthcare by re-examining clinical services in paramedicine. Prehosp Emerg Care 2017;21:652-61.

3 Health Quality Ontario. Under pressure: emergency department performance in Ontario, 2016. Available: http://www.hqontario.ca/ Portals/0/documents/system-performance/under-pressure-report-en. pdf

4 Institute of Medicine Committee on the Future of Emergency Care in the U.S. Health System. The future of emergency care in the United States health system. Ann Emerg Med 2006;48:115-20.

5 Jones A, Schumacher C, Bronskill SE, et al. The association between home care visits and same-day emergency department use: a casecrossover study. Can Med Assoc J 2018;190:E525-31 http://www. cmaj.ca/lookup/doi/

6 van Weel C, Kidd MR. Why strengthening primary health care is essential to achieving universal health coverage. CMAJ 2018;190:E463-6 https://www.cmaj.ca/content/190/15/E463

7 Gonçalves-Bradley D, Khangura JK. Primary care professionals providing non-urgent care in hospital emergency departments. Cochrane Database of Systematic Reviews 2018;2 https://www. cochranelibrary.com/cdsr/doi/10.1002/14651858.CD002097.pub4/ full

8 Eastwood K, Morgans A, Smith K, et al. Secondary triage in prehospital emergency ambulance services: a systematic review. Emerg Med J 2015;32:486-92 https://emj.bmj.com/lookup/doi/

9 Morgan SR, Chang AM, Alqatari M, et al. Non-Emergency department interventions to reduce ED utilization: a systematic review. Acad Emerg Med 2013;20:969-85 https://www.ncbi.nlm.nih. gov/pmc/articles/PMC4038086/

10 Morris J, Daoust R, Cournoyer A, et al. LO65: safety and satisfaction of a new program Redirecting low-acuity emergency department patients to medical clinic: a prospective cohort study. CJEM 2018;20:S29-30. doi:10.1017/cem.2018.127

11 Kirkland SW, Soleimani A, Rowe BH, et al. A systematic review examining the impact of Redirecting low-acuity patients seeking emergency department care: is the juice worth the squeeze? Emerg Med J 2019;36:97-106.

12 Schull MJ, Lazier K, Vermeulen M, et al. Emergency department contributors to ambulance diversion: a quantitative analysis. Ann Emerg Med 2003;41:467-76.

13 Purdy S, Griffin T, Salisbury C, et al. Ambulatory care sensitive conditions: terminology and disease coding need to be more specific to aid policy makers and clinicians. Public Health 2009;123:169-73. doi:10.1016/j.puhe.2008.11.001

14 Nagree Y, Camarda VJ, Fatovich DM, et al. Quantifying the proportion of general practice and low-acuity patients in the emergency department. Med J Aust 2013;198:612-5.

15 Gruneir A, Bell CM, Bronskill SE. Frequency and pattern of emergency department visits by long-term care Residents-A population-based study: ED visits by long-term care residents. J Am Geriatr Soc 2010:58:510-7.

16 Strum RP, Tavares W, Worster A. Establishing consensus on emergency department interventions that could be conducted in subacute care settings for nonemergent paramedic transported visits: a RAND/UCLA modified Delphi study. CMAJ Open 2021.

17 von Elm E, Altman DG, Egger M, et al. Strengthening the reporting of observational studies in epidemiology (STROBE) statement: guidelines for reporting observational studies. BMJ 2007;335:806-8.

18 Test. National ambulatory care reporting system. Toronto: Ontario: IC/ ES Central, 2021. 
19 Li G, Lau JT, McCarthy ML, et al. Emergency department utilization in the United States and Ontario, Canada. Acad Emerg Med 2007;14:582-4.

20 Okoli C, Pawlowski SD. The Delphi method as a research tool: an example, design considerations and applications. Inf Manage 2004;42:15-29. doi:10.1016/j.im.2003.11.002

21 Bennett C, Vakil N, Bergman J, et al. Consensus statements for management of Barrett's dysplasia and early-stage esophageal adenocarcinoma, based on a Delphi process. Gastroenterology 2012:143:336-46.

22 Wood L, Bjarnason GA, Black PC, et al. Using the Delphi technique to improve clinical outcomes through the development of quality indicators in renal cell carcinoma. J Oncol Pract 2013;9:e262-7. doi:10.1200/JOP.2012.000870

23 Bullard MJ, Unger B, Spence J, et al. Revisions to the Canadian emergency department triage and acuity scale (CTAS) adult guidelines. CJEM 2008;10:136-42. doi:10.1017/S1481803500009854
24 Canadian Institute for Health Information. Canadian coding standards for version 2018 ICD-10-CA and CCI. CIHI 2018

25 Durant E, Fahimi J. Factors associated with ambulance use among patients with low-acuity conditions. Prehosp Emerg Care 2012;16:329-37. doi:10.3109/10903127.2012.670688

26 Eastwood K, Morgans A, Smith K. Characteristics associated with emergency department suitability in low-acuity ambulance cases. Australasian Journal of Paramedicine 2020;17. doi:10.33151/ajp.17.782

27 Uscher-Pines L, Pines J, Kellermann A. Deciding to visit the emergency department for non-urgent conditions: a systematic review of the literature. Am J Manag Care 2013;19:47-59.

28 Ministry of Health. Ontario expanding innovative models to deliver better, connected care. news.ontario.ca. Available: https://news. ontario.ca/en/release/1000003/ontario-expanding-innovativemodels-to-deliver-better-connected-care 